\title{
Association between suicidal behaviour and impaired glucose metabolism in depressive disorders
}

\author{
Hannu Koponen ${ }^{1 *}$, Hannu Kautiainen ${ }^{2,3}$, Esa Leppänen ${ }^{4}$, Pekka Mäntyselkä ${ }^{2,5}$ and Mauno Vanhala ${ }^{2,3,6}$
}

\begin{abstract}
Background: Disturbances in lipid metabolism have been linked to suicidal behaviour, but little is known about the association between suicide risk and abnormal glucose metabolism in depression. Hyperglycaemia and hyperinsulinaemia may increase the risk of depression and also the risk for suicide, we therefore studied associations between suicidal behaviour and disturbances in glucose metabolism in depressive patients who had been referred to depression nurse case managers.
\end{abstract}

Methods: Patients aged 35 years and older ( $N=448$, mean age 51 years) who were experiencing a new depressive episode, who were referred to depression nurse case managers in 2008-2009 and who scored $\geq 10$ on the Beck Depression Inventory were enrolled in this study. The study was conducted in municipalities within the Central Finland Hospital District (catchment area of 274000 inhabitants) as part of the Finnish Depression and Metabolic Syndrome in Adults study. The patients' psychiatric diagnoses and suicidal behaviour were confirmed by the Mini-International Neuropsychiatric Interview. Blood samples, for glucose and lipid determinations, were drawn from participants after $12 \mathrm{~h}$ of fasting, which was followed by a 2-hour oral glucose tolerance test (OGTT) when blood was drawn at 0 and 2 h. Insulin resistance was measured by the Quantitative Insulin Sensitivity Check Index (QUICKI) method.

Results: Suicidal ideation (49\%) and previous suicide attempts (16\%) were common in patients with major depressive disorder or dysthymia. Patients with depression and suicidal behaviour had higher blood glucose concentrations at baseline and at 2 hours in the OGTT. Glucose levels associated positively with the prevalence of suicidal behaviour, and the linearity was significant at baseline ( $p$ for linearity: 0.012, adjusted for age and sex) and for 2-hour OGTT glucose ( $p$ for linearity: 0.004, adjusted for age and sex). QUICKI levels associated with suicidal behavior ( $p$ for linearity across tertiles of QUICKI: 0.026). Total and LDL cholesterol and triglyceride levels were also higher in those patients with suicidal behaviour. Multivariate analysis revealed that blood glucose levels, BDI scores and antidepressive medications associated with suicidal behaviour.

Conclusion: Insulin resistance and disturbances in glucose and lipid metabolism may be more common in middleaged depressive patients with suicidal behaviour.

Keywords: Cholesterol, Depression, Glucose, Insulin resistance, Suicidal behaviour, Ideation, Suicidality, Suicide attempt, Triglyceride

\footnotetext{
* Correspondence: hannu.j.koponen@hus.fi

${ }^{1}$ Old Age Psychiatry, University of Helsinki, and Helsinki University Hospital,

P.O. Box 22FIN-00014 Helsinki, Finland

Full list of author information is available at the end of the article
} 


\section{Background}

Depression is thought to have multifaceted pathophysiology and increased risk for having depression is related to type two diabetes and other cardiometabolic disorders [1-3]. Numerous studies have also documented an association between suicide risk and depression $[4,5]$. Increased suicide risk in depression may be related to various psychosocial factors, interpersonal conflicts and negative life events [6]. However, recent studies have reported an association between diabetes, depressive disorders and suicidal ideation $[7,8]$, in addition to an association between higher high glycated haemoglobin (HbA1c) values and suicidal ideation in subjects with diabetes [9]. Another cardiometabolic risk factor, disturbances in lipid metabolism, may also associate with impulsiveness and suicidal behaviour [10].

The monoamine hypothesis states that depression is related to monoaminergic dysfunction [11]. Reduced serotonin levels associate with increased weight, increased waist circumference, elevated blood glucose levels and insulin resistance in addition to depressed mood [12]. The inflammatory hypothesis of depression emphasizes the role of pro-inflammatory cytokines and cell-mediated immune activation that results in tryptophan depletion and serotonergic hypofunction [13-16]. Type 2 diabetes is recognized as an immune-mediated disease in which cytokines play an important role that leads to impaired insulin signaling and to the selective destruction of the insulinproducing beta cells [17]. A possible underlying pathophysiological mechanism for the emergence of abnormal glucose metabolism in depression and suicidal behaviour could thus lie in the interplay between serotonin and proinflammatory cytokines [18-20].

Studies on the associations between depression, suicidal behaviour, glucose levels or insulin resistance are scarce, although some previous studies suggest that higher glucose levels are associated with dysthymia [7] and higher HbA1c concentrations with recurrent or psychotic depression [7]. Bot and co-workers reported an association between suicidal ideation and HbA1c levels in diabetic subjects [9]. A recent meta-analysis reported an association between depression and insulin resistance [21]. The role of insulin secretion is more obscure, though a longitudinal study found that low insulin secretion is a risk factor for depression in middle-aged women [22]. Hyperglycaemia and insulin resistance may increase the risk of depression and also the risk of suicide, thus, we decided to study the associations between suicidal behaviour and disturbances in glucose metabolism in depressive patients that had been referred to depression nurse case managers.

\section{Methods}

Patients who were 35 years of age or older, who were having a new depressive episode, who were referred to depression nurse case managers in 2008-2009 and who also scored $\geq 10$ on the Beck Depression Inventory (BDI) [23] were enrolled in this study. The study (Finnish Depression and Metabolic Syndrome in Adults (FDMSA) study) was conducted in both urban and rural municipalities that were part of the Central Finland Hospital District, Finland with a geographically unified catchment area of 274 000 inhabitants. Enrolment was based on written and oral patient information. Each enrolled patient gave her/his written consent. The Ethics Committee of Central Finland Hospital District had approved the study protocol prior to the commencement of the study. Depression and type two diabetes are more prevalent in the Finnish population after 35 years of age, therefore we decided to focus on the $\geq 35$ year-old age group. The age limit of 35 years was also chosen to obtain a stable study population, which facilitates having a more prolonged follow-up. We obtained a representative sample comprising both mild and more severely depressive participants, by including self-referred patients along with general practitioner referred patients. In Finland, the depression nurse case managers are an important part of the basic health care team and they primarily organize the treatment of depressed patients together with the general practitioners. The patients were treated according to the current Finnish treatment guidelines for depression, i.e., with antidepressive medications and/or psychotherapy. If suicidal behaviour was a serious current problem, then those suicidal patients were further referred to a psychiatric hospital.

All participants completed a standard questionnaire that contained questions about their use of medications, which inter alia included antidepressant use and also hormone replacement therapy in females. In addition, data were collected on smoking habits, alcohol consumption (number of drinks per week) and physical activity (number of over-30-min exercise sessions per week). The severity of depressive symptoms was ascertained by scoring the responses to the 21-item BDI [23], which all patients had to complete and the psychiatric diagnosis was subsequently confirmed upon having a diagnostic interview. The diagnostic interview was the Mini-International Neuropsychiatric Interview (M.I.N.I.) [24]. The M.I.N.I. is a structured diagnostic interview that is compatible with the International Statistical Classification of Diseases and Related Health Problems, Tenth Revision (ICD-10), and the Diagnostic and Statistical Manual of Mental Disorders, Fourth Edition (DSMIV). Suicidal behaviour was defined in the present study according to the M.I.N.I. suicidal behaviour module as 'suicidal ideation during the past month' or 'suicide attempt during the past month or in a lifetime'. A total of 706 referred patients scored 10 or more for the BDI. However, only 448 patients were selected for the study 
population after having the diagnostic interview after which they were deemed to have met the criteria for major depressive disorder or for dysthymia.

Fasting blood samples were drawn between 8 and 11 o'clock after $12 \mathrm{~h}$ of fasting for glucose and lipid level determinations. This was followed by a 2-hour oral glucose tolerance test at 0 and $2 \mathrm{~h}$ sampling (OGTT). The physical examination included the participants' weight, height, waist circumference and blood pressure measurements, which were taken during the same study visit. Height and weight of the participants were determined with the participants wearing light clothing and was accurate to the nearest $0.5 \mathrm{~cm}$ and $0.1 \mathrm{~kg}$, respectively. The waist circumference was measured to the nearest $1.0 \mathrm{~cm}$ at the midpoint between the lateral iliac crest and the lowest rib. Trained nurses measured blood pressure twice with a mercury sphygmomanometer while patients/participant who had first been rested for $15 \mathrm{~min}$ were in a sitting position.

Modular Analytics SWA (Hitachi High-Technologies Corporation, Tokyo, Japan) served to determine serum total cholesterol, HDL cholesterol, LDL cholesterol, triglycerides and plasma glucose. The concentrations of serum insulin were analysed by a Siemens Advia Centaur (Siemens Health Care Diagnostics, Dublin, Ireland). We applied all the routine quality control procedures of an accredited clinical laboratory (Central Finland Hospital District, Clinical laboratory) including regular testing of standard samples. The sensitivity of the insulin assay was $2.4 \mathrm{U} / \mathrm{l}$, and the intra- and inter-assay coefficients of variation were $5.3 \%$ and $7.6 \%$, respectively. Insulin resistance may co-occur with depression, therefore we also calculated the Quantitative Insulin Sensitivity Check Index (QUICKI) on the basis of the insulin and glucose levels according to the equation: QUICKI $=1 /$ $[\log (\mathrm{I} 0)+\log (\mathrm{G} 0)]$, in which I0 is fasting insulin, and G0 is fasting glucose [25]. In QUICKI, low values are associated with insulin resistance.

\section{Statistical analysis}

The results are presented as means, interquartile ranges, standard deviations and frequency distributions. We used the Student's $t$-test, Mann-Whitney- $U$-test and the chi-square test to test for statistical significance between groups. The multivariate logistic regression model was used to investigate factors related to suicidal behaviour, and clinically relevant variables without covariability were selected from Table 1 for the procedure. We then used generalized linear models with the appropriate distribution and link functions to evaluate the statistical significance for the hypotheses of linearity. Any violations of the assumptions (non-normality), were evaluated by a bootstrap-type test. The normality of the variables was tested by using the Shapiro-Wilk W test.

\section{Results}

Nearly half 218 (49\%) of the 448 participants had suicidal thoughts, whereas 72 (16\%) of participants also had previously attempted suicide according to the results of the M.I.N.I. interview. Participants with depression and suicidal behaviour had higher blood glucose levels at baseline and at 2 hours with the OGTT (Table 1).

The blood glucose concentrations associated positively with the prevalence of suicidal behaviour (Fig. 1), and linearity was significant for baseline glucose concentrations ( $\mathrm{p}$ for linearity: 0.012, adjusted for age and sex) and for 2-hour OGTT glucose concentrations ( $\mathrm{p}$ for linearity: 0.004, adjusted for age and sex adjusted). The OR for suicidal behaviour for the highest vs. the lowest tertile of fasting glucose concentrations at baseline for the OGTT was 2.18 (95 \% CI: 1.27-3.74) whereas the corresponding tertile values for 2-hour glucose, OR was 1.99 (95 \% CI: 1.64-3.38). The baseline or 2-hour insulin levels were not significantly different from each other, but QUICKI levels showed a significant association with suicidal behaviour ( $\mathrm{p}$ for linearity across tertiles of QUICKI: 0.026; Fig. 2). When the 72 patients who had a previous suicide attempt were scrutinized separately from the 218 patients who had suicidal ideation, no statistically significant linearity in the OGTT values was observed.

Of the other cardiometabolic risk factors, total and LDL cholesterol and triglyceride levels were higher among participants who had either suicidal ideation or who had previously attempted suicide (Table 1). Patients with suicidal behaviopur also smoked more frequently, and alcohol dependence was also more common (Table 1). In the multivariate analysis, plasma glucose levels, BDI score and antidepressive medication associated with suicidal behaviour. No associations with suicidal behaviour and age, prevalence of type 2 diabetes, gender distribution, smoking, alcohol use disorder, or chronic diseases were found (Table 2).

\section{Discussion}

A novel finding in our study was the observed association between higher plasma glucose/blood glucose levels, insulin resistance and suicidal behaviour in depression. Nearly half of the participants in our study reported suicidal ideation or previous attempts at suicide, which were as frequent as those reported in another recent Finnish study, the Vantaa Depression Study [26]. Cases with a history of suicidal behaviour also had higher triglyceride concentrations in addition to elevated total and LDL cholesterol levels. Statins may slightly increase the risk for developing diabetes [27], but as the use of statins in our study was more prevalent in the subgroup with no suicidal behaviour our finding cannot be therefore attributed to statin medication. The 
Table 1 Groups taking part in the FDMSA study

\begin{tabular}{|c|c|c|c|}
\hline & No suicidal ideation and behaviour $N=230$ & Suicidal ideation and attempts in M.IN.I. $N=218$ & $p$ \\
\hline Women, n (\%) & $169(74)$ & $143(66)$ & 0.059 \\
\hline Age, mean (SD) & $51(10)$ & $51(10)$ & 0.64 \\
\hline Age $65+$ years old, $n$ (\%) & $22(10)$ & $22(10)$ & 0.66 \\
\hline Education years, mean (SD) & $11.2(2.9)$ & $11.2(2.8)$ & 0.95 \\
\hline Working status, n (\%) & & & 0.15 \\
\hline Employed & $108(47)$ & $81(37)$ & \\
\hline Retired & $61(27)$ & $62(28)$ & \\
\hline Unemployed & $56(24)$ & $69(32)$ & \\
\hline Student & $4(2)$ & $6(3)$ & \\
\hline Household income, 1000€, median (IQR) & $24(12,40)$ & $20(10,35)$ & 0.50 \\
\hline BMI, mean (SD) & $27.9(5.8)$ & $28.2(5.9)$ & 0.49 \\
\hline \multicolumn{4}{|l|}{ Blood pressure $(\mathrm{mmHg})$, mean (SD) } \\
\hline Systole & $130(16)$ & $131(15)$ & 0.45 \\
\hline Diastole & $82(11)$ & $82(11)$ & 0.90 \\
\hline \multicolumn{4}{|l|}{ Plasma Glucose, mmol/l, mean (SD) } \\
\hline $\mathrm{Oh}$ & $5.71(1.17)$ & $6.07(1.68)$ & 0.013 \\
\hline $2 \mathrm{~h}$ & $5.85(1.83)$ & $6.69(3.01)$ & 0.0013 \\
\hline Insu0, U/I, mean (SD) & $9.65(8.08)$ & $10.97(8.38)$ & 0.11 \\
\hline Insu1, U/I, mean (SD) & $58.5(58.9)$ & $69.3(69.9)$ & 0.12 \\
\hline QUICKI, mean (SD) & $0.16(0.01)$ & $0.15(0.01)$ & 0.023 \\
\hline Total cholesterol (mmol/l), mean (SD) & $4.97(0,98)$ & $5.22(1.06)$ & 0.017 \\
\hline HDL cholesterol (mmol/l), mean (SD) & $1.61(0.50)$ & $1.53(0.45)$ & 0.089 \\
\hline LDL cholesterol (mmol/l), mean (SD) & $2.90(0.83)$ & $3.21(1.00)$ & $<0.001$ \\
\hline Triglyceride (mmol/l), mean (SD) & $1.29(0.77)$ & $1.48(0.92)$ & 0.025 \\
\hline BDI score, mean (SD) & $21(7)$ & $26(8)$ & $<0.001$ \\
\hline median (IQR) & $20(15,25)$ & $26(20,32)$ & \\
\hline Smoking, n (\%) & $56(24)$ & $86(39)$ & $<0.001$ \\
\hline Coronary heart disease, n (\%) & $12(5)$ & $9(4)$ & 0.58 \\
\hline Arterial hypertension, n (\%) & $69(30)$ & $64(29)$ & 0.86 \\
\hline Previous stroke, n (\%) & $7(3)$ & $0(0)$ & 0.015 \\
\hline T2D, n (\%) & $30(13)$ & $24(11)$ & 0.50 \\
\hline Alcohol use disorder, n (\%) & $21(9)$ & $39(18)$ & 0.007 \\
\hline Antidepressive medication, n (\%) & $139(61)$ & $170(78)$ & $<0.001$ \\
\hline Statin medication, n (\%) & $57(25)$ & $39(18)$ & 0.007 \\
\hline Leisure time physical activity, n (\%) & & & 0.824 \\
\hline Low & $79(35)$ & $71(33)$ & \\
\hline Moderate & $56(25)$ & $50(24)$ & \\
\hline High & $91(40)$ & $92(43)$ & \\
\hline
\end{tabular}

T2D type 2 diabetes, BMI body mass index, QUICKI quantitative insulin sensitivity check index, Insu0 baseline serum insulin level, Insu1 serum insulin level after 2-hour OGTT, $B D I$ beck depression inventory, IQR interquartile range

multivariate analysis revealed that plasma glucose levels, BDI scores and antidepressive medications positively associated with suicidal behaviour. No associations with suicidal behaviour and age, prevalence of type 2 diabetes, gender distribution, smoking, alcohol use disorder, or chronic diseases were found however. With the exception of hypertension, cardiovascular diseases (CVDs) were rare due to the comparatively low age of the study population. Although a recent study found an association between obesity and suicidal behaviour [28], our 

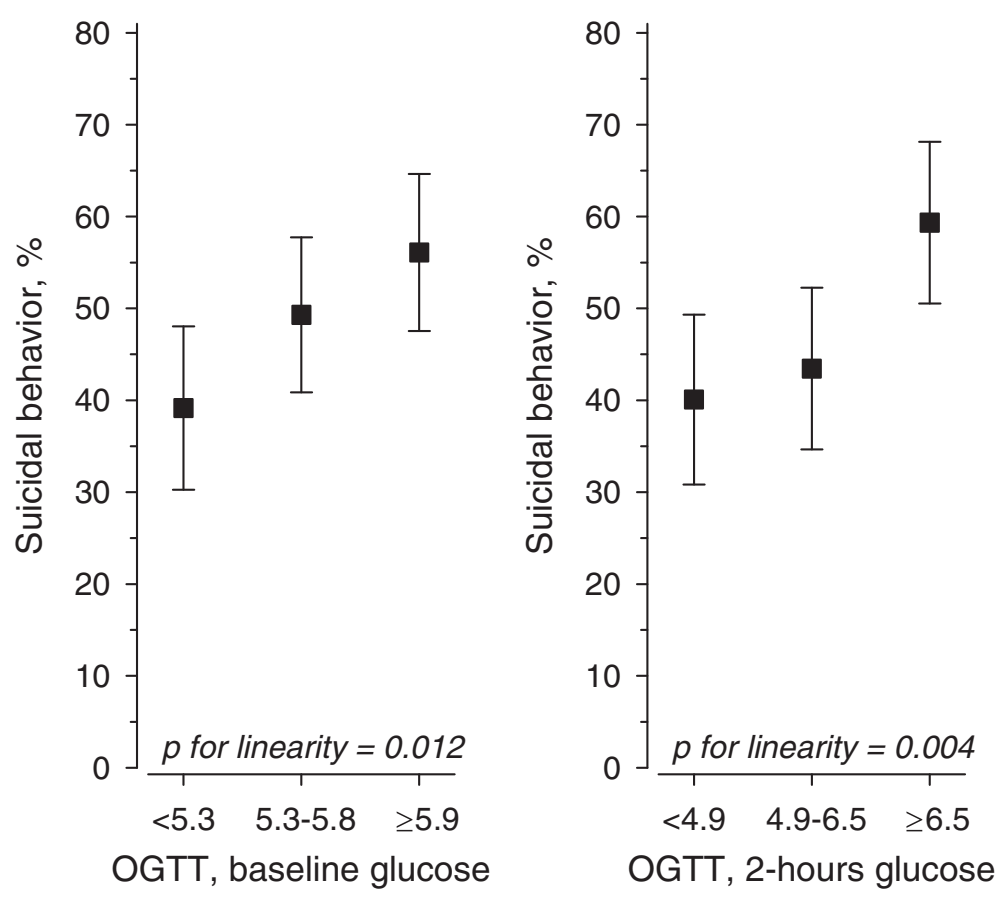

Fig. 1 Age and sex-adjusted association between baseline and 2-hour OGTT glucose tertiles and suicidal behaviour

study population showed no differences in body mass index levels.

We observed higher blood glucose for the OGTT both at baseline and at 2-hour sampling in patients with suicidal ideation or who had previously attempted suicide. Ceretta et al. [7] reported three-fold higher suicidal ideation rates in insulin-treated type 2 diabetic patients, which suggested an association between suicidal tendencies and blood glucose levels. Type 2 diabetes was more common in the subgroup with no suicidal behaviour in our study and this suggests that the observed association cannot solely be due to type 2 diabetes. Bot and coworkers [9] also reported an association between higher HbA1c values and depressive mood, sleep disturbance, appetite problems and suicidal ideation. Their study population comprised participants with either type 1 or type 2 diabetes, and the associations were more profound in type 1 diabetes [9]. Han and co-workers have also reported an increased risk for suicidal ideation in adults with diabetes and depression [8], but none of the previous studies have specifically studied the association between glucose levels and suicidal tendencies behaviour in depressed patients.

The association observed between glucose levels and suicidal behaviour may be related to the cytokinemediated inflammatory process that results in the activation of indoleamine 2,3-dioxygenase, the depletion of tryptophan and suicidal behaviour related to emerging serotonergic hypofunction, depression and impulsivity $[13,17,29,30]$. The groups in the present study showed no differences in baseline and 2-hour insulin levels, which suggests insulin resistance rather than insufficient insulin secretion was the probable associated factor. In addition, the surrogate index for insulin resistance, QUICKI, showed a significant association with suicidal behaviour, which lends further support for the association between disturbed glucose metabolism and suicidal behaviour. However, the patients with suicidal behaviour in our study also had higher BDI scores, so the severity of depression and the higher prevalence of alcohol use disorder in this group of patients may also have contributed to suicidal behaviour [31, 32]. In addition, hyperactivation of the hypothalamic-pituitary-adrenal axis has been reported in 20 to $80 \%$ of depressed individuals [33]. This hyperactivity has been put forward as an important mechanism that explains both the pathophysiology of depression and also its relationship with medical conditions such as diabetes and obesity. Higher cortisol levels are associated with age and more severe forms of depression, such as melancholic and psychotic depression, and may thus also contribute to the association between higher BDI levels and risk of suicidal behavior [33, 34].

Previous studies have identified smoking as a possible risk factor for attempted and also completed suicide [35]. Smoking has been linked to serotonergic dysfunction [3]. Serum cholesterol has been suggested to reflect the serotonergic function of the central nervous system and to participate in cognitive functioning in addition to mood regulation [10]. Low cholesterol levels have been 


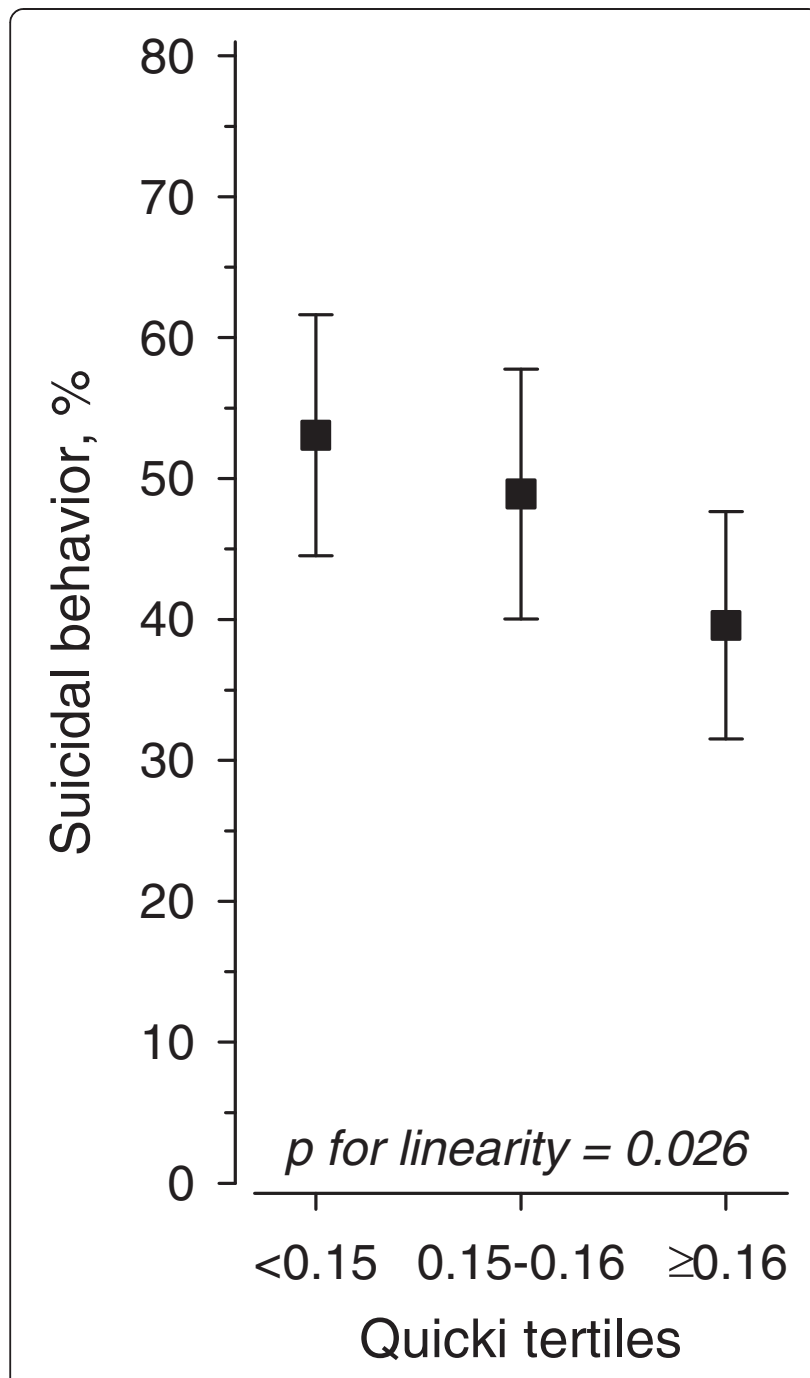

Fig. 2 Age and sex-adjusted association between QUICKI tertiles and suicidal behavior

linked by some studies to impulsiveness and the risk of suicide in depressive patients [10,36,37]. However, another study has reported that high cholesterol [38] concentrations to be associated with suicidal behaviour. In this study, we found higher total and higher LDL cholesterol levels in participants with suicidal behaviour, which is in line with the results of Silic et al. [18], who also reported an association between low platelet serotonin, and high interleukin IL-6, and glucose. The higher triglyceride levels were probably related to the insulin resistance or unhealthy lifestyles that were more common in the subgroup with suicidal behaviour [29].

The strengths of our study include its extensive and geographically representative sample of middle-aged and elderly subjects. We also used a diagnostic interview to complement the self-rating of the depressive symptoms. However, we cannot draw inferences about the causality
Table 2 Associations between suicidal behaviour and patient characteristics

\begin{tabular}{|c|c|c|}
\hline & OR $(95 \% \mathrm{Cl})$ & $P$-value \\
\hline Men & 1.58 (0.91 to 2.76$)$ & 0.10 \\
\hline Age & 0.99 (0.95 to 1.02$)$ & 0.36 \\
\hline Education years & 1.03 (0.95 to 1.12$)$ & 0.44 \\
\hline \multicolumn{3}{|l|}{ Working status } \\
\hline Employed & 1 (Reference) & \\
\hline Retired & 2.07 (1.04 to 4.11$)$ & 0.038 \\
\hline Un-employed & 1.22 (0.69 to 2.16$)$ & 0.49 \\
\hline Student & $1.34(0.25$ to 7.14$)$ & 0.74 \\
\hline BMI & $1.02(0.97$ to 1.06$)$ & 0.49 \\
\hline \multicolumn{3}{|l|}{ Plasma Glucose* tertiles } \\
\hline I $(\leq 5.2)$ & 1 (Reference) & 0.008 (linearity) \\
\hline \| (5.3 to 5.8$)$ & 1.33 (0.74 to 2.41$)$ & \\
\hline III ( $(\geq 5.9)$ & 2.50 (1.26 to 4.94$)$ & \\
\hline BDI score & $1.10(1.06$ to 1.13$)$ & $<0.001$ \\
\hline Smoking & 1.66 (0.99 to 2.76$)$ & 0.051 \\
\hline Coronary heart disease & $1.32(0.43$ to 4.10$)$ & 0.63 \\
\hline Arterial hypertension & 0.84 (0.45 to 1.55$)$ & 0.57 \\
\hline T2D & 0.69 (0.31 to 1.57$)$ & 0.38 \\
\hline Alcohol use disorder & 0.81 (0.39 to 1.68$)$ & 0.57 \\
\hline Antidepressive medication & 2.15 (1.30 to 3.54$)$ & 0.003 \\
\hline Statin medication & 0.55 (0.29 to 1.05$)$ & 0.071 \\
\hline \multicolumn{3}{|l|}{ Leisure time physical activity } \\
\hline Low & 1 (Reference) & 0.45 (linearity) \\
\hline Moderate & 0.77 (0.43 to 1.39$)$ & \\
\hline High & 0.81 (0.48 to 1.37$)$ & \\
\hline
\end{tabular}

due to the cross-sectional design of our study, which is a limitation. In addition, only persons aged 35 or older were enrolled in the study, so the results cannot be generalized to younger age groups. The evaluation of insulin resistance by QUICKI relying on the mathematical modelling of fasting plasma glucose and insulin concentration may also be a limitation, although a recent study reported that QUICKI results were comparable to a euglycemic hyperinsulinemic clamp and superior to measurements based on insulin alone [39].

\section{Conclusions}

The observed association between plasma glucose levels, insulin resistance and suicidal behaviour suggests that disturbances in glucose metabolism are associated with suicidal ideation and attempts. However, further studies are necessary to elucidate the pathophysiology behind these associations, and it remains obscure whether the treatment of cardiometabolic risk factors lowers the risk of suicidal behaviour. 


\section{Competing interests}

The authors declare that they have no competing interests.

\section{Authors' contributions}

HKo participated the study design and co-ordination and wrote the manuscript; HKa, contributed substantially to the acquisition of data, performed the statistical analyses, and contributed substantially to the drafting of the manuscript; EL contributed substantially to the acquisition of the laboratory data and participated in the drafting of the manuscript; PM participated in the study design and co-ordination and contributed substantially to the drafting of the manuscript; MV participated in the study design and co-ordination and contributed substantially to the drafting of the manuscript. All authors contributed to and have approved the final manuscript.

\section{Acknowledgements}

The authors thank the following nurse case managers, who took part in the practical implementation of the FDMSA: Mari Alanko, Harri Back, Timo Hannula, Anu Holopainen, Ritva Häkkinen, Katja Johansson, Eija Kinnunen, Kaija Luoma, Hannele Niemi, Hillevi Peura, Inga Pöntiö, Kirsi Rouvinen, Tiina Silvennoinen and Marianne Vihtamäki, as well as FDMSA study nurses Anne Kirmanen, Reetta Oksanen and Olli Niemi, and Pia Jauhiainen, scientific secretary of the study.

\section{Author details}

${ }^{1}$ Old Age Psychiatry, University of Helsinki, and Helsinki University Hospital, P.O. Box 22FIN-00014 Helsinki, Finland. ${ }^{2}$ Primary Health Care Unit, Kuopio University Hospital, Kuopio, Finland. ${ }^{3}$ Primary Health Care Unit, Central Hospital of Central Finland, Jyväskylä, Finland. ${ }^{4}$ Public Utility Laboratory KESLAB, Central Finland Hospital District, Jyväskylä, Finland. ${ }^{5}$ Primary Health Care Unit, Institute of Public Health and Clinical Nutrition, School of Medicine, University of Eastern Finland, Kuopio, Finland. ${ }^{6}$ Department of Health Sciences, University of Eastern Finland, Kuopio, Finland.

Received: 8 April 2015 Accepted: 16 July 2015

Published online: 22 July 2015

\section{References}

1. Ayuso-Mateos JL, Vasquez-Barquero JL, Dowrick C, Lehtinen V, Dalgard OS, Casey $\mathrm{P}$, et al. Depressive disorders in Europe: prevalence figures from the ODIN study. Br J Psychiatry. 2001;179:308-16.

2. Kessler RC, McGonagle KA, Zhao S, Nelson CB, Hughes M, Eshleman S, et al. Lifetime and 12-month prevalence of DSM-III-R psychiatric disorders in the United States. Results from the National Comorbidity Survey. Arch Gen Psychiatry. 1994;51:8-19.

3. Kessler RC, Berglund P, Demler O, Jin R, Koretz D, Merikangas KR, et al. The epidemiology of major depressive disorder: results from the National Comorbidity Survey Replication (NCS-R). JAMA. 2003;289:3095-105.

4. Isometsä E, Henriksson M, Marttunen M, Heikkinen M, Aro H, Kuoppasalmi K, et al. Mental disorders in young and middle aged men who commit suicide. BMJ. 1995:310:1366-7.

5. Bertolote JM, Fleischman A, De Leo D, Wasserman D. Suicide and mental disorders: do we know enough? Br J Psychiatry. 2003;183:382-3.

6. Van Reedt Dortland AKB, Vreeburg SA, Giltay EJ, Licht CMM, Vogelzangs N, van Veen $T$, et al. The impact of stress systems and lifestyle on dyslipidemia and obesity in anxiety and depression. Psychoneuroendocrinology. 2013;38:209-18.

7. Ceretta LB, Reus GZ, Abelaira HM, Jordana LK, Schwalm MT, Hoepers NJ, et al. Increased prevalence of mood disorders and suicidal ideation in type 2 diabetic patients. Acta Diabetol. 2012;49 suppl 1:S227-34.

8. Han SJ, Kim HJ, Choi YJ, Lee KW, Kim DJ. Increased risk of suicidal ideation in Korean adults with both diabetes and depression. Diabetes Res Clin Pract. 2013;101:14-7.

9. Bot M, Pouwer F, de Jonge P, Tack JC, Geelhoed-Duijvestin PHLM, Snoek FJ. Differential associations between depressive symptoms and glycemic control in outpatients with diabetes. Diabet Med. 2013;30:e115-22.

10. Troisi A. Low cholesterol is a risk factor for attentional impulsivity in patients with mood symptoms. Psychiatry Res. 2011;188:83-7.

11. Stahl SM. Stahl's Essential Psychopharmacology. Neuroscientific Basis and Practical Applications. 3rd ed. Cambridge, UK: Cambridge University Press; 2008.

12. Muldoon MF, Mackey RH, Korytkowski MT, Flory JD, Pollock BG, Manuck SB. The metabolic syndrome is associated with reduced central serotonergic responsivity in healthy community volunteers. J Clin Endocrinol Metabolism. 2006;91:718-21.

13. Dunn AJ, Swiergiel AH, de Beaurepaire R. Cytokines as mediators of depression: What can we learn from animal studies? Neurosci Biobehav Rev. 2005;29:891-909.

14. Christmas DM, Potocar JP, Davies SJC. A biological pathway linking inflammation and depression: activation of indoleamine 2,3-dioxygenase. Neuropsychiatr Dis Treat. 2011;7:431-9.

15. Maes M. Depression is an inflammatory disease, but cell-mediated immune activation is the key component of depression. Progr NeuroPsychopharmacol Biol Psychiatry. 2011;35:664-75.

16. Maes $M$, Ringel K, Kubera M, Berk M, Rybakowski J. Increased autoimmune activity against 5-HT: A key component of depression that is associated with inflammation and activation of cell-mediated immunity, and with severity and staging of depression. J Affect Disord. 2012;136:386-92.

17. Banerjee M, Saxena M. Interleukin-1 (IL-1) family of cytokines: Role in Type 2 diabetes. Clinica Chimica Acta. 2012;413:1163-70.

18. Silic A, Karlovic D, Serretti A. Increased inflammation and lower platelet 5-HT in depression with metabolic syndrome. J Affect Disord. 2012;141:72-8.

19. Valkanova V, Ebmeier KP, Allan CL. CRP, IL-6 and depression: Asystematic review and meta-analysis of longitudinal studies. J Affect Disord. 2013;150:736-44.

20. Serafini G, Pompili M, Seretti ME, Stefani H, Palermo M, Coryell W, et al. The role of imflammatory cytokines in suicidal behavior: A systematic review. European Neuropsychopharmacol. 2013;23:1672-86.

21. Kan C, Silv N, Golden SH, Rajala U, Timonen M, Stahl D, et al. A systematic review and meta-analysis of the association between depression and insulin resistance. Diabetes Care. 2013;36:480-9.

22. Akbaraly TN, Kumari M, Head J, Ritchie K, Ancelin M-L, Tabak AG, et al. Glycemia, insulin resistance, insulin secretion, and risk of depressive symptoms in middle age. Diabetes Care. 2013;36:928-34.

23. Beck AT, Ward CH, Mendelson M, Mock J, Erbaugh J. An inventory for measuring depression. Arch Gen Psychiatry. 1961;4:561-71.

24. Sheehan DV, Lecrubier Y. Mini-International Neuropsychiatric Interview (MINI). Tampa, FL: University of South Florida, Institute for Research in Psychiatry; 1994. Paris: INSERM-Hopital de la Salpetriere.

25. Katz A, Nambi SS, Mather K, Baron AD, Follman DA, Sullivan G, et al. Quantitative insulin sensitivity check index: a simple, accurate method for assessing insulin sensitivity in humans. J Clin Endocrin Metab. 2000;85:2402-10.

26. Sokero PT, Melartin TK, Rytsälä HJ, Leskelä US, Lestelä-Mielonen PS, Isometsä ET. Suicidal ideation and attempts among psychiatric patients with major depressive disorder. J Clin Psychiatry. 2003;64:1094-100.

27. Sattar N, Preiss D, Murray HM, Welsh P, Buckley BM, de Craen AJM, et al. Statins and the risk of incident diabetes: a collaborative meta-analysis of randomized statin trials. Lancet. 2010;375:735-42.

28. Mather AA, Cox BJ, Enns MW, Sareen J. Associations of obesity with psychiatric disorders and suicidal behaviors in a nationally representative sample. J Psychosom Res. 2009;66:277-85.

29. Kressel G, Trunz B, Bub A, Hülsman O, Wolters M, Lichtinghagen R, et al. Systemic and vascular markers of inflammation in relation to metabolic syndrome and insulin resistance in adults with elevated atherosclerosis risk. Athetosclerosis. 2009;202:263-71.

30. Sublette ME, Galfalvy HC, Fuchs D, Lapidus M, Grunebaum MF, Oquendo MA, et al. Plasma kynurine levels are elevated in suicide attempters with major disorder. Brain Behav Immun. 2011;25:1272-8.

31. Suokas JT, Perälä J, Suominen K, Saari S, Lönnqvist J, Suvisaari JM. Epidemiology of suicide attempts among persons with psychotic disorder in the general population. Schizophrenia Res. 2010;124:22-8.

32. Mäntyselkä P, Korniloff K, Saaristo T, Koponen H, Eriksson J, Puolijoki H, et al. Association of depressive symptoms with impaired glucose regulation, screen-detected and previously known type 2 diabetes - findings from the Finnish D2D survey. Diabetes Care. 2011;34:71-6.

33. Stetler C, Miller GE. Depression and hypothalamic-pituitary-adrenal activation: A quantitative summary of four decades of research. Psychosomatic Med. 2011;73:114-26.

34. Belvedere Murri R, Pariante C, Mondelli V, Masotti M, Atti AR, Mellacqua Z, et al. HPA axis and aging in depression: Systematic review and meta-analysis. Psychoneuroendocrinology. 2014;41:46-62.

35. Malone KM, Waternaux C, Haas GL, Cooper TB, Li S, Mann JJ. Cigarette smoking, suicidal behavior, and serotonin function in major psychiatric disorders. Am J Psychiatry. 2003;160:773-9. 
36. Olie E, Picot MC, Guillaume S, Abbar M, Courtet P. Measurement of total serum cholesterol in the evaluation of suicidal risk. J Affect Disord. 2011;133:234-8.

37. Tedders SH, Fokong KD, McKenzie LE, Wesley C, Yu L, Zhang J. Low cholesterol is associated with depression among US household population. J Affect Disord. 2011:135:115-21. 51: 1060-1063.

38. Tanskanen A, Vartiainen E, Tuomilehto J, Viinamäki H, Lehtonen J, Puska P. High serum cholesterol and risk of suicide. Am J Psychiatry. 2000;157:648-50.

39. Mather K, Hunt AE, Steinberg HO, Paradisi G, Hook G, Katz A, et al. Repeatability characteristics of simple indices of insulin resistance: Implications for research applications. J Clin Endocrinol Metabolism. 2001;86:5457-64.

\section{Submit your next manuscript to BioMed Central} and take full advantage of:

- Convenient online submission

- Thorough peer review

- No space constraints or color figure charges

- Immediate publication on acceptance

- Inclusion in PubMed, CAS, Scopus and Google Scholar

- Research which is freely available for redistribution 